\title{
Multiobjective Optimization for Optimal Design of Urban Drainage Systems
}

\author{
Misgana K. Muleta and Paul F. Boulos
}

\begin{abstract}
Control of sewer overflows, the leading cause of water pollution in the nation's water bodies, is vital to reducing risks to public health and protecting the environment. The most common solutions for mitigating sewer overflows include adding storage volume, increasing conduit capacity, expanding pumping capacity, and implementation of real time operational controls to more effectively utilize existing system storage. Obviously, comprehensive modeling and analysis of these sewer systems becomes necessary for developing sound cost-effective and reliable solutions for enhancing system integrity and performance to convey sewer flows without causing overflows. However, identification of the optimal remedial solution that effectively circumvents overflow problems with the least expenditure is a daunting task. The current practice involves a tedious trial-and-error evaluation procedure that seldom leads to the most effective or most economical solutions. Another emerging design approach utilizes single objective optimization that identifies the solution that best satisfies a predefined criterion. The performance criterion used with single objective optimization subjectively lumps the economics objective with metrics that measure effectiveness of the remedial solution from the perspective of avoiding overflows (e.g., minimizing the number of flooding events or reducing the flooding volume). Consequently, the design solution identified using single objective optimization depends on the weights subjectively placed on the two incommensurable and conflicting objectives, and may not represent the global optimal solution. A preferable approach is to seek tradeoff solutions commonly referred to as non-dominated solutions or Pareto-optimal solutions. The methodology proposed here links an extended version of the EPA SWMM 5 model, a comprehensive drainage network simulator, with NSGA-II, an evolutionary multiobjective optimization method with a proven history of identifying Pareto-optimal solutions for a wide range of engineering problems. The method should prove useful to any wastewater utility attempting to improve system integrity, reliability and performance and optimize its capital improvement program.
\end{abstract}

\section{Introduction}

Control of sewer overflows is vital to reducing risks to public health and protecting the environment from water pollution. Sanitary sewer overflows (SSOs) and combined sewer overflows (CSOs) may release partially treated or untreated sewage to surface waters. This untreated sewage overflows contains microbial pathogens, suspended solids, toxics, nutrients, trash, and other pollutants causing serious water quality problems. Wet weather flows from rainfall-derived inflow and infiltration (RDII) can exceed capacity of the sewer pipes resulting in an SSO. Other causes of SSOs include blockage of the sewer conduits by grease and debris, sediments buildup, pipe breaks, leaking manholes, offset joints, equipment failures, undersized sewer pipes, power outages, and other factors. When an SSO occurs, sewage flows into streets, playgrounds and streams. This sewage 
can also back up into basements, causing property damage and creating threats to public health for those who come in contact with the untreated sewage.

CSOs occur in older combined sewer systems that were designed to carry both sanitary sewage and storm water runoff to a wastewater treatment plant. Under dry conditions, the wastewater treatment plant (WWTP) treats the sewage and then discharges it to a water body. During periods of heavy rainfall or snowmelt, however, the wet weather volume in the combined sewer system may exceed the available hydraulic capacity of the sewer system or treatment plant. This can lead to the discharge of excess wastewater directly to nearby streams, rivers, or other water bodies without treatment. Combined sewer systems in the United Sates serve roughly 746 communities serving about 40 million people. Although there are combined sewers in 32 states and the District of Columbia, they are mostly located in the Northeast and Great Lakes regions, and the Pacific Northwest (U.S. EPA, 2004).

With the growing expectations by the public for quality services, the Environmental Protection Agency (EPA) under the authority of the Clean Water Act adopted by Congress has implemented pollution control programs and set wastewater standards for the industry. In order to meet these requirements, comprehensive modeling and analysis of these sewer systems becomes necessary for developing sound cost-effective solutions for enhancing system integrity and performance to reliably convey sewer flows without causing overflows and backups.

Drainage network simulation models are commonly used to plan and design better sewer collection systems (Nicklow et al., 2004, 2006). System improvement alternatives commonly evaluated include the addition of new sewer pipes or treatment capacity, increasing conduit capacity (bigger interceptors), more storage volume, pumping capacity, and efficient utilization of existing storage in the system through implementation of real time operational controls. The daunting task is, however, to choose an improvement option or combination of options that effectively solve the flooding problem with the least capital expenditure. No single improvement approach can be generalized to every collection system. Performance and economic effectiveness of the remedial alternatives varies from system to system. The current practice of selecting an improvement option involves a tedious trial-and-error evaluation procedure that seldom leads to the most effective or most economical solution for upgrading collection systems. This procedure makes use of a drainage network simulation model to evaluate the hydraulic performance of the existing system for different design alternatives (modifications) under a range of loading and operating conditions. The design option that meets the target hydraulic criteria with the lowest cost is selected among the alternative designs. The complexity of this manual trial-and-error procedure increases exponentially with the number of proposed system modifications and corresponding operating conditions. It is important to point out that even if the target performance specifications are met, the trial-and-error procedure has no inherent feature that assures that the solution reached is cost optimum or even cost effective. Good engineering procedure dictates that the iterations continue until a number of promising alternatives have been evaluated. However, given the vast number of possible combinations of system enhancements, it is unlikely that even the most experienced modeler will be able to determine the least-cost improvement alternative using a trial-and-error procedure. Therefore, the result of using 
the traditional trial-and-error evaluation approach is often inefficient performance at a greater cost.

One way to circumvent the limitations of a trial-and-error procedure is to employ optimization theory. This paper presents an optimal design methodology that eliminates the need of the traditional manual design technique. The problem of choosing least-cost improvements for urban drainage systems is solved using optimization theory. The procedure consists of determining the optimal design improvement solution that produces the minimum overall cost while satisfying target system performance requirements. The decision variables can include any selected combination of pipe slope and upsizing, storage, pumping and new piping. Performance criteria include maximum allowable depth to diameter ratio, minimum and maximum conduit velocities, maximum head loss for force mains, and minimum and maximum conduit slopes. This gives practicing engineers complete control over the solution process.

The proposed approach links a comprehensive drainage network simulator with a multiobjective optimization model and iterates between the simulator and the optimization model until Pareto-optimal solutions are found. The optimization procedure employed is the Nondominated Sorting Genetic Algorithm II (NSGA-II) (Deb et al, 2002), which has been proven to effectively generate reliable solutions for multiobjective problems across wide areas of engineering applications. The urban drainage network simulation is performed using an extended version of the EPA storm water management model, SWMM 5 (Rossman, 2005). The optimization model generates improved sets of decision variables that seek to minimize design costs and maximize system performance.

\section{Multiobjective Optimization}

Most realistic engineering problems involve simultaneous optimization of several objectives that are often conflicting. In urban drainage systems, for example, the objective is to improve system performance to avoid flooding with the least possible cost. Obviously, improvement solutions that utilize large pipe sizes, storage volumes, or pump capacities can easily achieve the target performance criterion by avoiding or minimizing overflows. However, these solutions will perform very poorly from the economics perspective since they demand large capital expenditure. Thus, these two objectives (i.e. improving performance to avoid flooding and minimizing implementation cost) are conflicting in the sense that solutions that improve either objective may do so at the expense of degrading the other objective. This is a typical behavior of realistic multiobjective optimization problems (MOPS), which makes them significantly different from single-objective optimization problems.

In single-objective optimization, an optimal solution is usually unique and clearly defined. However, the typical goal in multiobjective optimization is to find satisfactory trade-offs between competing objectives. These trade-off solutions are referred to as nondominated solutions. A solution is said to be Pareto optimal if it is not dominated by any other solution when all objectives are taken into consideration. Various methods exist for multiobjective optimization. Recently, evolutionary algorithms (EAs) have become established as an alternative to the traditional methods of simple aggregation (see Srinivas and Deb, 1994; Zitzler and Thiele, 1999). The main advantages of EAs based MOPs include their ability to search large decision spaces, thus raising the likelihood of 
locating the global Pareto-optimal set, and their capability to generate multiple trade-offs within a single optimization, unlike aggregation methods that require multiple optimization runs. In EAs, the only significant difference between single objective and multiobjective evaluations is the way of assigning fitness value to a solution candidate so that the fitness measure accurately reflects the performance of the alternative solution relative to its counterparts. In single objective optimization, the objective function value itself can be used as a measure of fitness. However, in multiobjective evaluations, it is necessary to design a means of converting the multidimensional objective functions into a scalar fitness measure. Based on the technique used to map the multiple performance values to a single fitness value (i.e., the fitness assignment approach), there are a wide variety of EA based methods for solving MOPs (Fonseca and Fleming, 2000).

NSGA-II was selected for this study based on the comparative study results conducted by Kunzli et al. (2004) and Khare (2002). These studies compared a number of multiobjective algorithms and concluded that NSGA-II (Deb et. al, 2002) and Strength Pareto Evolutionary Algorithm (SPEA 2) (Zitzler et al., 2001) exhibit the best overall performance. Furthermore, NSGA-II was found to be the most computationally efficient among the algorithms considered. NSGA-II incorporates a non-dominating sorting approach, a feature that distinguished NSGA-II as the fastest multi-objective evolutionary algorithm (Khare, 2002). NSGA-II uses the crowded-comparison operator to maintain diversity along the Pareto optimal front. Search for optimal Pareto front using NSGA-II begins with random generation of potential solutions of size $N$. These initial candidates, known as parent population, are sorted based on the concept of Pareto dominance (Veldhuizen and Lamont, 2000), and each solution is assigned a fitness value equal to its non-domination level (i.e., 1 corresponds to the best non-domination level, 2 is the next best level, and so on). Then, an offspring population of the same size as the parent population (i.e., $N$ ) is created using binary tournament selection, recombination, and mutation operators. Search procedure on subsequent generations involves combining the offspring with the parent population and conducting nondominated sorting that systematically groups the solutions into different nondominated fronts based on the concept of Pareto dominance and optimality (i.e., best non-dominated solutions, the second best, and so on). Solution alternatives for the next generation are chosen from the nondominated fronts in descending order until $N$ members are obtained. If the first nondominated front contains solutions less than $N$, the remaining solutions will be taken from the second nondominated front, and so on. However, the last front to contribute solution members to the next generation may have to be sorted using the crowdedcomparison operator. The crowded-comparison operator is the average distance between an individual solution and those solutions nearest to it in the objective space. Once all the $N$ members of the parent population for the next generation are chosen, a new offspring population is produced using binary tournament selection based on the crowdingcomparison operator, crossover, and mutation operators. The same procedure is repeated until convergence criteria have been met. For further detail on NSGA-II, the reader is referred to Deb et al. (2002). 


\section{Urban Drainage Simulation Model}

InfoSWMM (Boulos, 2005), the urban drainage simulation model used in this study, utilizes an extended version of the EPA SWMM5 (Rossman, 2005) to solve urban drainage hydrology, hydraulics and water quality. InfoSWMM can model the entire land phase of the hydrologic cycle (i.e., surface runoff, vadose zone processes, groundwater flow, evaporation, and snow fall and snow melt) as applied to urban stormwater and wastewater collection systems. The model can perform single event or long-term (continuous) rainfall-runoff simulations accounting for spatial and/or temporal variability in climate, soil, land use, and topographic conditions of the urban watershed. Rainfallrunoff simulations could be conducted using several approaches including the non-linear reservoir method, Colorado Urban Hydrograph Procedure (CUHP), Santa Barbara hydrograph method, the Modified Rational Formula, and various synthetic unit hydrograph approaches including the Natural Resources Conservation Service (NRCS) methods, Delmarva, Snyder, Clark, and Espey methods. In addition to runoff quantity, InfoSWMM can simulate runoff quality including buildup and washoff of pollutants from primarily urban watersheds composed of developed and non-developed land uses.

Once runoff quantity and quality are simulated and wastewater loads at receiving junctions are determined, the routing portion of InfoSWMM can transport the flow using either steady, kinematic wave or dynamic wave routing, through a conveyance system of pipes, channels, storage/treatment devices, pumps, and hydraulic regulators such as weirs, orifices, and other outlet types. The model can accurately simulate flow conditions of any level of complexity including those experiencing backwater effect, flow reversal, and pressurized flow. Because it simultaneously solves the solution for both water levels at nodes and flows in conduits, the model can be applied to any general network layout, even those containing multiple downstream diversions and loops. The model also offers advanced Real-Time Control (RTC) scheme for the operational management of hydraulic structures.

InfoSWMM is a complete decision support system embedding intrinsic modeling and optimization capabilities in GIS. It explicitly integrates ESRI ArcGIS geospatial model with the extended version of SWMM5 and other modules including automated dry weather flow generation and allocation, automated subcatchment delineation and parameter extraction, automatic model calibration, an optimization capability for screening design alternatives to address every facet of urban drainage infrastructure management, a sophisticated tool that dynamically determines storage in gravity conduits, an advanced pond design module. The GIS interface allows seamless communication between the GIS and the various modeling applications. It provides the geospatial platform for developing and calibrating network models, screening and analyzing design alternatives, and reporting and displaying results. The resulting decision support system effortlessly reads GIS datasets, extracts necessary modeling information, and automatically constructs, loads, designs, calibrates, analyzes and optimizes a representative urban drainage models considering hydrologic, hydraulic, and water quality performance requirements. It also makes it easy to run, simulate and compare various modeling scenarios, identify system deficiencies, and determine cost-effective physical and operational improvements to achieve optimum performance and regulatory 
compliance. These combined capabilities provide a consistent environment to plan, design, and operate safe and reliable urban drainage systems.

\section{Problem Formulation}

The goal of the multiobjective urban drainage formulation is to identify the "best" combination of conduit sizes, storage volumes, and pumping capacities needed for one or more facilities in the collection system that meet target system performance requirements (i.e., avoid flooding) at minimum cost. The objective functions of the optimization problem consist of minimizing both the overall design cost and overflow volume under a specified set of loading and operating conditions. They can be mathematically expressed as:

Minimize $\quad \sum_{c=1}^{C} C_{c} L_{c}+\sum_{s=1}^{S} C_{s} V_{s}+\sum_{p=1}^{P} C_{p}$

Minimize $\quad \sum_{t=0}^{t=T} V_{t}+$ Penality

where $c, C_{c}$ and $L_{c}$ used in the left-hand side term of equation (1) refer to the number of conduits, the replacement cost which is a function of conduit size, and the length of the conduit, respectively. Likewise the $s, C_{s}$, and $V_{s}$ used in the middle term of equation (1) refer to storage node counter, cost per unit volume of storage and volume of the storage unit, respectively. The $p$ and $C_{p}$ used in the last term of equation (1) refer to pump counter and cost associated with the pump, respectively. The $V$ term in the second equation refers to overflow volume from all the facilities in the collection system at time $t$. Overall, equation (1) evaluates economic implication of the design solutions and equation (2) describes the performance of the design solution from the perspective of reducing overflow volume. The decision variables, which consist of conduit, storage and pump sizes, are automatically determined to minimize the objective functions while satisfying implicit system constraints, explicit bound constraints and explicit variable constraints. The implicit constraints on the sewer collection system are equality constraints defining the hydraulic equilibrium state of the system. They correspond to the conservation of mass and momentum equations (Saint Venant equations) that govern the unsteady flow of water through a drainage network of channels and pipes. These equations are solved implicitly using InfoSWMM, by converting them into an explicit set of finite difference formulas that compute the flow in each conduit and head at each node for time $t+\Delta t$ as functions of known values at time $t$.

\section{ExplicitC onstraints}

The explicit bound constraints represent system performance criteria and may include constraints on conduit flow velocity $(V)$, conduit slope $(S)$, and head loss $(H G)$ in force mains for a given set of sewer system loading and operating conditions. Conduit constraints describe hydraulic limits on the conduits. These limits include minimum and maximum velocities, maximum head losses for force mains, and maximum and minimum 
invert elevations. These limits are based on design criteria consistent with local regulations or engineering practice. The minimum velocity constraint ensures that a sufficient velocity is maintained to keep solids in suspension and to encourage passage of sewer floatables. The maximum velocity criterion is critical for pressurized mains to ensure that momentum forces are not objectionably high on the system especially at joints. For gravity mains, maximum velocity is usually not a significant issue; however, under some conditions, the transition from supercritical to subcritical flows can create a large hydraulic jump and may be of concern to the engineer. The maximum head loss constraint ensures that pressurized mains have sufficient lift to carry the water to the upstream location.

Occasionally local codes specify geometric requirements for gravity sewer systems, which typically include slope and embedment depth. For constructability purposes, a minimum slope (and inter alia velocity) must be maintained. The embedment depth requirement accommodates areas where freezing weather or surcharge surface loading (beneath railways or streets) is of concern. The invert elevation at the downstream end of each conduit (expressed as an offset from the node invert elevation) constrains the design to meet the slope and embedment depth requirements. When a design solution does not satisfy an implicit bound constraint, a penalty method is used to handle the constraint violation.

A penalty cost is added to the objective function to penalize an infeasible solution (degrade its fitness) and force the search procedure towards the region of feasible solutions. The penalty cost function is defined as the divergence (distance) of the computed solution from the feasible region or:

$$
P C=\sum_{i=1}^{N} v_{i}\left|C_{i}(x)-C_{i}\right|
$$

where $N$ represents the number of constraints; $v_{i}$ represents a weighting factor associated to constraint $C_{i}, C_{i}(x)$ is the value of the $i^{\text {th }}$ constraint and $C_{\mathrm{i}}$ is the constraint limit. It is expected that different values of the penalty costs will result in different solutions and also affect the efficacy of the optimization calculation. Therefore, a number of trial optimization runs with different penalty costs may be required to better explore the solution space and narrow in to the lowest cost solutions.

The explicit variable constraints are used to set minimum (lower) and maximum (upper) limits on the sewer sizes and to specify the discrete (commercially available) diameter values for the new conduits. Conduits should be lumped together in separate logical design groups based on their known physical characteristics such as size and location. As such, all conduits within a group will possess an identical size. For each conduit group, the conduit size is bound by an explicit inequality constraint as:

$$
\operatorname{Dmin}_{n} \leq D_{n} \leq \operatorname{Dmax}_{n} \quad \forall n, \forall D_{n} \in D^{0}=\left\{d_{k}^{0}, k=1, \ldots K\right\}
$$

where $\operatorname{Dmin}_{n}$ designates the lower bound (the minimum value) of conduit sizes for conduit group $n$; $\operatorname{Dmax}_{n}$ represents the upper bound (the maximum value) of conduit sizes for conduit group $n$; and $D_{n}$ is the conduit size for conduit group $n$ and selected from a set of available conduit sizes of $D^{0}$. Similarly, pump curves are selected from 
various user-specified pump curves to represent pump station expansion. Storage volumes are computed based on shape of storage, defined either as a mathematical function or in tabular form (head vs. area), and are bound by user-specified minimum and maximum storage depths.

\section{Solution Methodology}

The optimal urban drainage system design/rehabilitation problem formulated above is solved using a dual-level optimal control methodology that integrates SWMM5 drainage network simulator with NSGA-II, an evolutionary algorithm based multiobjective optimization method. Starting with an initial feasible set of decision variables, it is passed to InfoSWMM for use in explicitly satisfying the implicit system constraints and in evaluating the implicit bound constraints. The InfoSWMM solution is then passed back to the optimization model for use in quantifying the objective function and any violations in the implicit bound constraints. This information is then utilized to produce an improved set of decision variables that automatically satisfies the explicit variable constraints and that seeks to minimize the objective functions. This iterative process is repeated until the best solution is found. The resulting optimization model has been successfully applied to a number of actual sewer collection systems in the US. The results show that significant improvements in system performance can be realized.

\section{Conclusion}

Computer based network simulation models provide the most viable and effective means of evaluating urban drainage system performance, identifying deficiencies, and assessing the reliability of proposed design improvement alternatives to avoid sewer overflows and reduce risks to public health and protect the environment from water pollution. However, identification of improvement solutions that effectively circumvent overflow problems with least expenditure requires an optimal control methodology that integrates advanced multiobjective optimization tool with comprehensive urban drainage simulation model. Historically, most urban drainage model design/rehabilitation attempts have typically employed a manual trial-and-error approach. Such an iterative approach often proves time consuming and costly when dealing with most typical urban stormwater systems. In this paper, an improved design model is developed using multiobjective optimization theory. The optimization model computes the optimal combination of pipe upsizing and alignment, storage size, and pump capacity to best meet desired system performance requirements at lowest cost. NSGA-II is coupled with a network solver for use in determining optimal design solutions. The model is further integrated with a geographical information system to provide a consistent geospatial decision support system for use in developing and calibrating large and complex urban drainage system models, and optimizing them to achieve improved performance at maximum savings. The resulting decision support system should prove useful to any wastewater utility attempting to optimize its capital improvement program. 


\section{References}

Boulos, P.F. (2005) 'InfoSWMM Suite - Users Guide', MWH Soft, Inc., Pasadena, CA.

Deb, K., Pratap, A., Agarwal, S., and Meyarivan, T. (2002). "A Fast and Elitist Multiobjective Genetic Algorithm: NSGA-II.” IEEE Trans. on Evolutionary Computation, 6(2): 182-197.

Fonseca, C.M., and Fleming, P. J. (2000). "Multiobjective optimization.” In Back, T., Fogel, D.B., and Michalewicz, Z. (editors), Evolutionary Computation 2, Advanced Algorithms and Operators. Institute of Physics, Philadelphia, PA.

Khare, V. (2002). Performance Scaling of Multi-Objective Evolutionary Algorithms. M.Sc. Thesis, School of Computer Science, The University of Birmingham, Edgbaston, Birmingham, UK.

Kunzli, S., Bleuler, S., Thiele, L., and Zitzler, E. (2004). "A Computer Engineering Benchmark Application for Multiobjective Optimizers." in Applications of MultiObjective Evolutionary Algorithms, ed. By C.A. Coello Coello and G.B. Lamont, World Scientific Publishing, London.

Nicklow, J.W., Boulos, P.F., and Muleta, M.K., 'Comprehensive Sewer Collection Systems Analysis Handbook for Engineers and Planners', 1st ed., MWH Soft, Inc. Publ., Pasadena, CA, 2004, 273 pp.

Nicklow, J.W., Boulos, P.F., and Muleta, M.K., 'Comprehensive Sewer Collection Systems Analysis Handbook for Engineers and Planners', 1st ed., MWH Soft, Inc. Publ., Pasadena, CA, 2004, 273 pp.

Rossman, L.A. (2005). "Stormwater Management Model User's Manual, Version 5", U.S. EPA, Cincinnati, OH.

Srinivas N., Deb K. (1994). "Multiple Objective Optimizations using Nondominated Sorting in Genetic Algorithms." Evolutionary Computation, 2(2): 221-248.

U.S. EPA (2004). Report to Congress: Impacts and Control of CSOs and SSOs; U.S.EPA Office of Water; Washington, D.C.; August 2004.

Veldhuizen, D. A. V., \& Lamont, G. B. (2000). "Multiobjective Evolutionary Algorithms: Analyzing the state-of-the-art." Evolutionary Computation, MIT press, 8(2): 125-147.

Zitzler E., and Thiele L. (1999). "Multiobjective Evolutionary Algorithms: A Comparative Case Study and the Strength Pareto Approach." IEEE Transactions on Evolutionary Computation, 3(4): 257-271. 
Zitzler, E., Laumanns, M. and Thiele, L. (2001). SPEA2: Improving the Strength Pareto Evolutionary Algorithm, TIK-Report 103, Computer Engineering and Network Laboratory (TIK), Swiss Federal Institute of Tech., Zurich, Switzerland. 